\title{
Etude et développement d'un verre intelligent pour le suivi d'activité à domicile des patients post-AVC
}

\section{Study and development of a smart cup for the home-based activities} monitoring of post-stroke patients

\author{
Maxence Bobin ${ }^{1}$, Mehdi Boukallel ${ }^{2}$, Margarita Anastassova ${ }^{3}$, Mehdi Ammi ${ }^{4}$ \\ ${ }^{1}$ LIMSI - CNRS, Université Paris-Saclay, France, maxence.bobin@limsi.fr \\ ${ }^{2}$ CEA - LIST, Université Paris-Saclay, France, mehdi.boukallel@cea.fr \\ ${ }^{3}$ CEA - LIST, Université Paris-Saclay, France, margarita.anastassova@cea.fr \\ ${ }^{4}$ LIMSI - CNRS, Université Paris-Saclay, France, mehdi.ammi@limsi.fr
}

RÉSUMÉ. Dans ce papier, nous présentons une plateforme de suivi d'activité continu et de guidage des patients postAVC à domicile durant leurs activités de la vie quotidienne. La plateforme consiste en un verre intelligent qui embarque des capteurs afin de suivre l'activité du bras et de la main lors de la journée. Le verre détecte son orientation, le niveau d'eau, sa position par rapport à une cible spécifique ainsi que les tremblements. De plus, un affichage permet de guider le patient dans ses mouvements. Nous présentons également deux études cliniques prévues avec les thérapeutes et les patients.

ABSTRACT. In this paper, we present a platform for continuously monitoring and guiding post-stroke patients at home during their daily activities. The platform consists of a smart cup that carries sensors to monitor the arm and hand activity during the day. The cup detects its orientation, the water level, its position relative to a specific target as well as the hand tremors. In addition, display can guide the patient in his movements. We also present two planned clinical studies with therapists and patients.

MOTS-CLÉS. Accident Vasculaire Cérébral, Monitoring, A Domicile, Verre, Internet des Objets, Stroke, Internet of Things.

KEYWORDS. stroke, monitoring, home, smart, cup, internet of things.
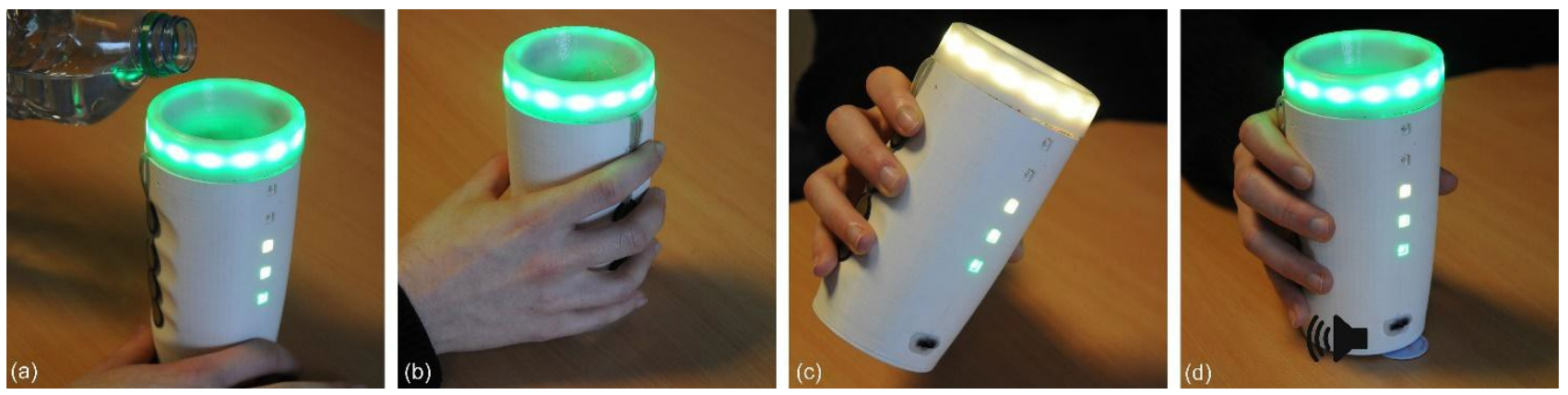

Figure 1. SyMPATHy lors des phases (a) de remplissage, (b) de saisie, (c) de déplacement et (d) de positionnement

\section{Introduction}

15 millions de personnes sont victimes d'un Accident Vasculaire Cérébral (AVC) chaque année. Les victimes d'AVC sont confrontées à divers déficits cognitifs et moteurs [1]. Parmi elles, 5 millions décèdent et 5 millions demeurent handicapées. Les handicaps post-AVC sont divers et varient en fonction de la zone du cerveau touchée. Des déficits moteurs comme la paralysie spasmodique ou la faiblesse musculaire et des troubles oculaires sont souvent observés [1], [2]. La rééducation post-AVC demeure chère en terme d'infrastructure et de personnel médical. De plus, aucun suivi à domicile ne permet d'évaluer l'évolution de l'état de santé des patients. En général, les progrès sont évalués avant 
chaque séance de rééducation par le thérapeute à l'aide de mesures empiriques basées sur des estimations visuelles [3].

Les nouvelles technologies de l'information et de la communication (NTICs) ainsi que l'Internet des Objets (IoT) fournissent des solutions flexibles pour le suivi d'activité. Cependant, ces plateformes se limitent à des exercices spécifiques en lien avec l'AVC ou requièrent la présence d'un thérapeute [4].

En outre, les méthodes classiques d'évaluation de l'AVC sont empiriques et basées sur des estimations visuelles qui ne peuvent être récupérées qu'au cours de la session d'évaluation avec les thérapeutes [5], [6]. Les objets intelligents sont des solutions prometteuses pour surmonter ces limitations et sont très adaptés au contexte familial [7], [8]. Plusieurs recherches ont mis en évidence de nouvelles méthodes d'évaluation impliquant des mesures objectives telles que les caractéristiques cinématiques des mouvements [9]-[12]. Ces mesures sont un outil efficace pour l'évaluation qualitative des patients atteints d'AVC, en particulier avec des objets intelligents. Enfin, certaines recherches ont mis en évidence la pertinence des activités de la vie quotidienne (AVQs) (cuisine, nettoyage, etc.) pour une évaluation continue et transparente des patients [13].

Pour surmonter les limites des plates-formes existantes et des approches de suivi d'activité postAVC pour la rééducation, nous proposons SyMPATHY, un verre intelligent qui intègre un réseau de capteurs permettant de surveiller l'activité des patients post-AVC à domicile. Le verre est un objet commun que les patients utilisent à différents moments de la journée (eau, café, etc.). Lorsque le verre est manipulé, plusieurs informations peuvent être récupérées telles que l'orientation du verre, le niveau du liquide, sa position relative par rapport à une cible spécifiée et l'évolution des tremblements. Le verre intelligent comprend également un affichage visuel pour fournir des informations de guidage gestuel utiles au patient lorsqu'il n'utilise pas ou ne manipule pas correctement le verre.

Ce papier présente la conception et l'implémentation d'un verre intelligent, appelé SyMPATHy, basé sur une AVQ : remplir un verre et boire. Le but est de suivre l'activité de la main et du bras chez un patient victime d'un AVC et de fournir un guidage durant les exercices de rééducation.

\section{Processus de conception}

Le processus de conception de SyMPATHy se décompose en cinq étapes et est basé sur la méthodologie suivante :

- Identification de

- la tâche à effectuer

- des informations à monitorer

- du feedback sensoriel à fournir au patient

- Implémentation de la plate-forme

- Acquisition des données et traitement

- Etude technique de la plateforme

- Etudes cliniques en prévision

\subsection{Tâche à effectuer}

L'objectif principal est de fournir aux patients un dispositif utile, utilisable et couramment utilisé. Afin d'identifier la tâche à effectuer, nous avons interrogé deux professionnels qualifiés travaillant dans un centre de rééducation. Les entretiens ont mis en évidence que les AVQs permettent de faire du suivi d'activité transparent, ce qui signifie que l'activité des patients dans la vie quotidienne ne sera pas impactée. De plus, Timmermans et al. a montré que le positionnement et la manipulation d'objets sont 
les tâches préférées des patients post-AVC [14]. Selon la littérature [15], [16] et les retours des professionnels, notre travail se concentre sur la tâche d'attraper, remplir et transporter un verre. Cette tâche se base sur différentes sous-tâches motrices (mouvement du bras, saisie avec la main, etc.) des membres supérieurs, également impliqués dans d'autres AVQs (ménage, prendre une douche, etc.). De plus, cette tâche implique simultanément un feedback visuel, tactile, proprioceptif et audio.

\subsection{Informations à monitorer}

La tâche à effectuer étant choisie (manipulation du verre), nous avons identifié les données à récolter durant l'utilisation du verre. Les entretiens ont soulignécinq principales données permettant d'évaluer le rétablissement du patient :

- Le niveau d'eau lors du remplissage

- L'orientation du verre durant la manipulation

- La position relative du verre par rapport à une référence

- L'apparition et l'évolution de tremblements

- La pression exercée sur le verre

Le suivi du niveau du liquide permet d'analyser la vitesse de remplissage ainsi que la quantité de liquide versée dans le verre. Cette information sera utilisée par le thérapeute pour évaluer la précision et la coordination des mouvements pendant le remplissage. En réalité, verser de l'eau dans un verre est un véritable défi pour les patients souffrant d'AVC et de déficits moteurs. Le patient doit saisir la bouteille et le verre, soulever la bouteille au-dessus du verre et contrôler la quantité de liquide versée dans le verre. Le suivi de l'orientation du verre permet au thérapeute de comprendre comment le patient a tenu le verre (verticalement ou non). Cela pourrait mettre en évidence certains troubles moteurs ou neurologiques. Etudier la position relative du verre lorsqu'il est placé sur la table permet au thérapeute d'évaluer la précision motrice du patient. Le thérapeute indique généralement au patient une cible à atteindre sur la table. Ensuite, la pression appliquée sur le verre lors de la saisie est un bon indicateur de la dextérité des patients. Enfin, le suivi de l'évolution de la fréquence des tremblements ainsi que leur puissance est un bon indicateur du rétablissement du patient [17]. Il permet également la détection de l'apparition de troubles neurologiques.

\subsection{Choix des feedbacks sensoriels}

Le feedback sensoriel fait référence à un signal qui permet au patient de savoir s'il effectue une tâche correctement ou non. Fournir des informations d'alerte pendant le remplissage et sur l'orientationdu verre lorsque le patient boit améliore leurs performances et leur motivation. En effet, plusieurs recherches ont mis en évidence l'impact direct des retours positifs ou négatifs sur la motivation des patients [18].

Avec la plate-forme SyMPATHy, nous proposons au patient des informations sur (1) le niveau du liquide, (2) l'orientation du verre et (3) la position relative du verre par rapport à une cible spatiale. Le tremblement n'a pas été pris en considération puisque le malade ne peut pas agir dessus. Il en est de même pour la pression appliquée sur le verre lors de la saisie. L'identification de modalités sensorielles appropriées et la pertinence de la représentation de l'information sont importantes pour guider les patients atteints d'AVC.

Premièrement, le feedback sélectionné pour l'orientation du verre est un feedback visuel pour mettre en évidence l'angle d'inclinaison et la direction. En effet, le retour audio ne peut pas fournir facilement des informations d'angle et de direction. D'un point de vue technique, nous avons utilisé un cercle de LEDs placé sur le dessus du verre. La couleur des LEDs varie selon l'angle d'inclinaison du verre. Nous avons sélectionné des couleurs discriminables (vert, rouge, etc.) pour afficher les niveaux 
d'inclinaison afin d'éviter une ambiguïté de perception. Les LEDs sont allumés en vert si le verre est maintenu correctement $\left(0-20^{\circ}\right)$, rouge si le verre est très incliné $\left(>50^{\circ}\right)$ et jaune ou orange pour les configurations intermédiaires (jaune: $20-35^{\circ}$, orange: $35-50^{\circ}$ ). Comme le matériau utilisé pour concevoir le verre est translucide, la lumière des LEDs est suffisamment diffusée pour éviter les problèmes d'occultation lorsque le verre est incliné.

Deuxièmement, une étude informelle nous a conduits à utiliser la modalité visuelle en affichant les couleurs verticalement le long du verre pour le niveau de liquide. Les couleurs simplifient l'affichage des informations en fournissant une représentation discrète du niveau de liquide. D'un point de vue technique, nous avons utilisé une colonne de 5 LEDs. Selon la culture européenne, nous avons allumé les LEDs du rouge en bas au vert en haut, en passant par l'orange et le jaune.

Troisièmement, en ce qui concerne la position du verre, nous nous sommes concentréssur « atteindre une cible spatiale prédéfinie ». Pour indiquer au patient que la cible est atteinte, nous avons utilisé un retour audio binaire. Cela permet de décharger la modalité visuelle. Du point de vue technique, un haut-parleur a été intégré au verre. Il joue une tonalité lorsque le patient apporte le verre sur la cible souhaitée.

\section{Implémentation de la plate-forme}

\subsection{Données collectées}

Le prototype SyMPATHY intègre des capteursqui permettentd'accéder aux données requises : (1) orientation du verre, (2) niveau du liquide, (3) position relation par rapport à une cible donnée, (4) détection des tremblements et (5) la pression appliquée sur le verre lors de la saisie. Tous ces capteurs sont reliés à un Raspberry Pi Zéro embarquant un noyau Linux complet.

\subsubsection{Orientation du verre}

Nous avons utilisé une centrale inertielle (IMU) pour mesurer le mouvement et l'orientation du verre. Nous avons choisi la centrale inertielle à 9 axes "Invensense MPU-9150". Elle présente un bon compromis entre performance, efficacité énergétique, taille etcoût. L'Invensense MPU-9150 intègre un accéléromètre, un gyroscope et un magnétomètre. Chacun de ces capteurs renvoie 3 valeurs sur les 3 axes $\mathrm{x}, \mathrm{y}, \mathrm{z}$.

\begin{tabular}{|c|c|c|}
\hline Capteur & Valeur & FSR \\
\hline Accéléromètre & Accélération & $\pm 2 \mathrm{~m} . \mathrm{s}-2(\mathrm{~g})$ \\
\hline Gyroscope & Vitesse angulaire & $\pm 1000^{\circ} . \mathrm{s}-1$ \\
\hline Magnétomètre & Champ magnétique & $\pm 1200 \mu \mathrm{T}$ \\
\hline
\end{tabular}

Tableau 1. Spécification de la centrale inertielle

FSR (Full-Scale Range) mappe les données brutes des registres de [-215, 215+1] à [ \pm Valeur FSR]. A $25^{\circ} \mathrm{C}$, le gyroscope présente une tolérance au facteur d'échelle de sensibilité de $3 \%$. La tolérance initiale d'étalonnage de l'accéléromètre est égale à $3 \%$. De plus, l'échantillonnage des données s'effectue à $30 \mathrm{~Hz}$ afin d'avoir un affichage dynamique fluide. 


\subsubsection{Niveau d'eau}

En raison des contraintes des capteurs de niveau de liquide industriels (faible réactivité, taille, etc.), nous avons conçu un capteur spécifique pour SyMPATHy. Le capteur est basé sur la conductivité du liquide. Nous avons placé cinq électrodes conductrices verticalement à l'intérieur du verre afin d'obtenir cinq niveaux différents. Les électrodes sont espacées d'un centimètre en hauteur. Chaque niveau discret correspond à un volume de $100 \mathrm{ml}$. Les électrodes ont été reliées à des ponts diviseurs de tension pour mesurer les tensions électriques lorsque le liquide ferme le circuit. Après des mesures empiriques avec des liquides de natures différentes, les résistances ont été fixées à $100 \mathrm{~K} \Omega$ à l'exception de la première qui a été fixée à $200 \mathrm{~K} \Omega$. Ces valeurs permettent de détecter la présence ou l'absence de liquide.

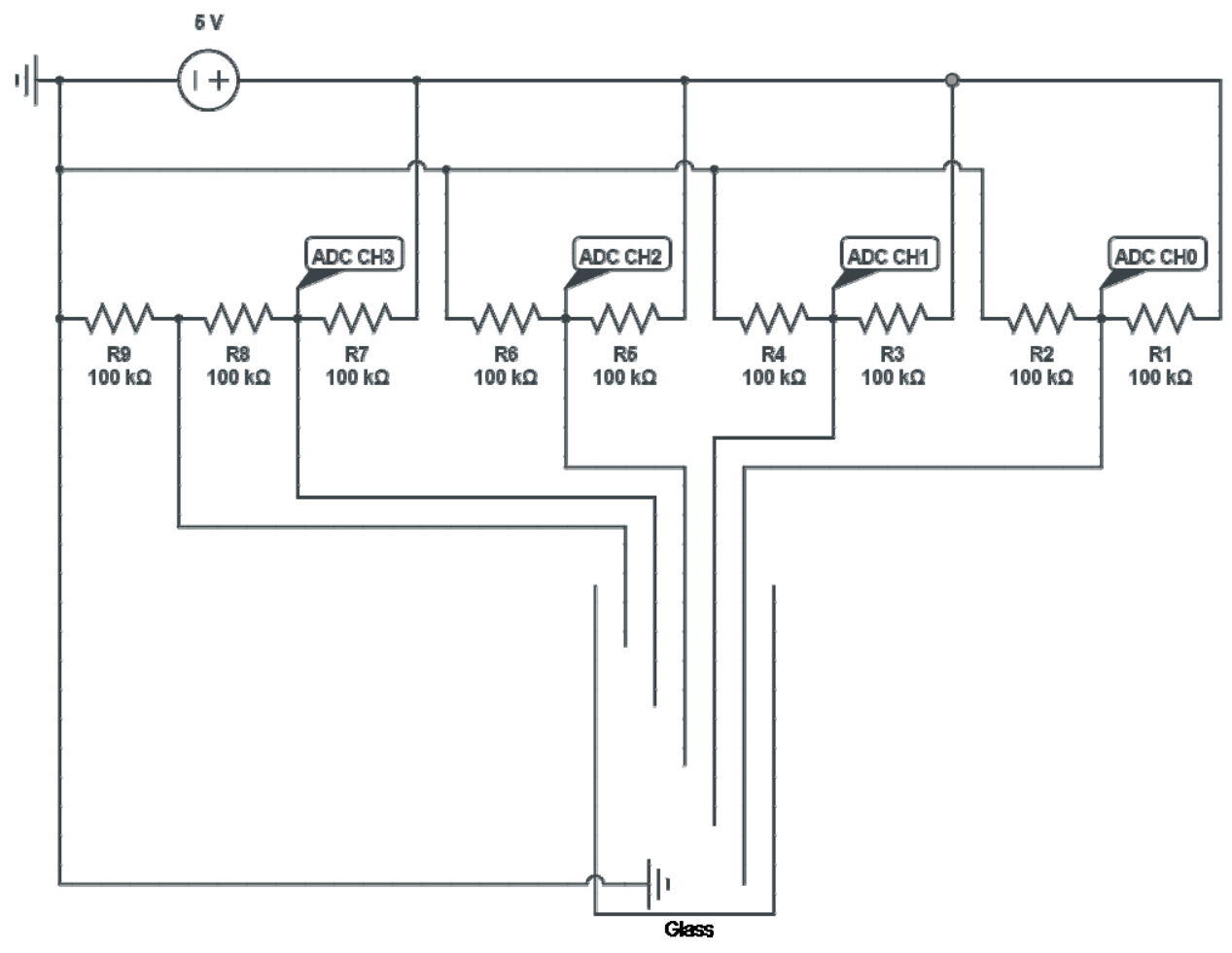

Figure 2. Schéma électronique du capteur de niveau de liquide

\subsubsection{Position relative du verre}

Pour détecter si le patient atteint correctement la cible positionnée sur la table, nous avons équipé le prototype SyMPATHY d'un lecteur NFC (Near Field Communication) et utilisé unTag NFCcomme cible. Le lecteur NFC détecte très rapidement le Tag NFC et donc si le verre atteint la cible. Nous avons utilisé un contrôleur PN532 NFC. La forme de l'antenne est un rectangle de 45 x 55 mm ce qui signifie que le Tag NFC peut être détecté dans une plage de $25 \mathrm{~mm}$ à $30 \mathrm{~mm}$ sur le plan horizontal. La distance minimale de détection verticale est d'environ $10 \mathrm{~mm}$. Nous avons utilisé un Tag Mifare classique qui fournit un identifiant de 7 octets.

\subsubsection{Détection des tremblements}

Du point de vue technique, les tremblements correspondent à des oscillations de faible amplitude. Des travaux antérieurs ont mis en évidence que les tremblements post-AVC ont une fréquence inférieure à $5 \mathrm{~Hz}$ et sont perpendiculaires à la direction du mouvement [19]. Sur la base de cette découverte, nous avons utilisé les trois axes du gyroscope pour mesurer les tremblements. Un traitement est appliqué sur les données extraites pour détecter et caractériser les tremblements (voir section "Traitement des données"). 
Des capteurs de pressions appelés Force-Sensitive Resistor (FSR) ont été ajoutés sur le verre. Ils permettent de remonter à la pression appliquée par chaque doigt de la main sur le verre. La résistance de chaque capteur varie d'une valeur infinie lorsqu'aucune pression n'est appliquée à une résistance de $10.25 \mathrm{~K} \Omega$ lorsqu'une force de 100 Newtons est appliquée. Cependant, ces capteurs ne permettent que d'avoir une approximation de la pression appliquée par le patient sur le verre. En effet, ils ne sont pas conçus pour effectuer des mesures précises. Il serait intéressant d'utiliser d'autres types de capteurs nous permettant de retracer une carte des pressions appliquées en tout point du verre.

\subsection{Conception du modèle 3D du verre}

La conception du verre a présenté plusieurs défis. Tout d'abord, le verre doit être étanche. Nous avons utilisé une imprimante 3D et nous avons testé l'étanchéité du verre avec des valeurs de remplissage différentes. Le paramètre optimal pour avoir un verre étanche avec un temps d'impression raisonnable est une valeur de remplissage de 30\%. Deuxièmement, pour intégrer la partie électronique, un petit espace est présent dans la partie inférieure du verre ainsi que des socles sur les parois internes. Le Raspberry Pi Zéro ainsi que les boosters de tension et le capteur de niveau de liquide sont fixés sur les parois internes. La batterie, le chargeur, l'IMU, le lecteur NFC, le haut-parleur et le dongle Wifi sont placés dans le compartiment inférieur. Le lecteur NFC a été placé au fond de cet espace (fixé sur la base de verre) afin qu'il puisse détecter lesTags NFC sur la table. Les électrodes utilisées pour détecter le niveau de liquide sont reliées aux ponts diviseurs de tensions situés dans le fond du verre. En ce qui concerne les affichages, nous avons utilisé des bandes de LEDs. Une bande de LEDs est placée en cercle sur le dessus du verre pour indiquer l'inclinaison de verre. Une autre bande de cinq LEDs est placée sur le côté du verre pour afficher le niveau de liquide. Enfin, afin d'aider le patient à saisir le verre, nous avons ajouté une empreinte de main sur le verre. En effet, l'empreinte de main fournit une information pertinente au patient sur la façon de saisir le verre [20]. Cette accessibilité est particulièrement utile pour les patients atteints d'AVC.

\section{Acquisition et traitement des données}

\subsection{Calcul embarqué et consommation énergétique}

La communication sans fil en temps réel (Bluetooth et Wi-Fi) avec un ordinateur a été envisagée pour enregistrer et traiter les données. Cependant, cette solution présente plusieurs contraintes en termes de facilité d'utilisation et de fiabilité de la communication. Par exemple, la communication Bluetooth nécessite une courte distance du point d'accès limitant l'espace de travail. En outre, la communication Bluetooth peut induire une perte de données rendant la plate-forme moins fiable pour le suivi des patients. La contrainte principale d'une communication sans fil en temps réel est la consommation d'énergie. Ce type de communication est un processus très gourmand ce qui représente un défi majeur dans le domaine de l'Internet des Objets. Pour résoudre ce problème avec la plate-forme SyMPATHY, nous avons enregistré les données localement dans des fichiers. Les données enregistrées sont envoyées à l'ordinateur une fois par jour ou une fois par semaine permettant au thérapeute de visualiser l'historique des données. Pour la plate-forme SyMPATHy, nous avons utilisé une batterie de $3.7 \mathrm{~V}$ et $2500 \mathrm{mAh}$. Les composants les plus consommateurs d'énergie sont les LEDs qui nécessitent 1140mA (60 mA x19) pour être allumées. L'autonomie de SyMPATHY est d'environ une journée avec l'éclairage, et d'une semaine sans l'éclairage.

\subsection{Algorithme de fusion pour détecter l'orientation du verre}

La centrale inertielle renvoie neuf valeurs d'accélération, de vitesse angulaire et de champ magnétique. Sur la base de ces valeurs, nous calculons la pose du capteur, autrement dit l'orientation du verre dans l'espace. Le framework RTIMULib-Arduino, de richards-tech, a été utilisé pour extraire les données de l'IMU et appliquer l'algorithme de fusion. RTIMULib utilise l'algorithme de (c) 2017 ISTE OpenScience - Published by ISTE Ltd.London, UK-openscience.frPage | 6 
fusion RTQF, une version simplifiée d'un filtre de Kalman pour une fusion efficace des données. RTQF utilise les mesures gyroscopiques avec le temps entre les échantillons pour extrapoler linéairement à partir de l'orientation précédente, l'orientation actuelle prédite de l'IMU. Afin de maintenir cette prédiction correcte, l'accéléromètre et le magnétomètre fournissent une référence absolue (tangage et roulis pour l'accéléromètre, lacet pour le magnétomètre). L'algorithme RTQF calcule deux quaternions à chaque étape: un quaternion prédit à partir des mesures gyroscopiques et un quaternion mesuré référencé par un cadre au sol à partir de l'accéléromètre et du magnétomètre. Le quaternion prédit est stable mais sujet à la dérive tandis que le quaternion mesuré est moins stable mais n'a aucune dérive. Slerp (Interpolation linéaire sphérique) est une technique pour trouver un quaternion intermédiaire entre deux autres quaternions. Le coefficient de Slerp (entre 0 et 1) contrôle vers lequel(le quaternion prédit ou le quaternion calculé) tends le quaternion résultant. Si la valeur est 0 , le quaternion mesuré est ignoré et seul le gyroscope est utilisé efficacement. Si la valeur est 1 , le quaternion prédit est ignoré et seul l'état mesuré à partir des accéléromètres et des magnétomètres est utilisé. Nous avons mis le coefficient Slerp à une valeur de 0,02 . Il en résulte que la fusion fonctionne très bien. Même si les mesures de gyroscope et d'accéléromètre dépassent leur FSR par le mouvement, l'algorithme prendra quelques secondes pour combler l'écart entre l'état prédit et l'état mesuré. RTIMULib permet également d'appliquer un filtre passe-bas (LPF) afin de supprimer les bruits. Nous avons ajouté un LPF avec une fréquence de coupure de $20 \mathrm{~Hz}$ pour le gyroscope et $21 \mathrm{~Hz}$ pour l'accéléromètre. Ces fréquences de coupure ont été sélectionnées après des mesures empiriques. En effet, comme les tremblements de l'AVC sont inférieurs à $5 \mathrm{~Hz}$ [19], les données de signal supérieures à $20 \mathrm{~Hz}$ ne sont pas nécessaires.

L'algorithme de fusion renvoie un quaternion qui correspond à l'orientation du verre dans l'espace. Sur la base de ce quaternion, il est possible de calculer la couleur des LEDs (vert, jaune, orange, rouge, blanc) pour fournir le feedback visuel aux patients. L'angle entre l'inclinaison du verre et le vecteur vertical $(0,0,1)$ dans le repère de référenceterrestre est exprimé encoordonnées sphériques. Selon le quaternion de pose [qx, qy, qz, qw], nous avons créé la matrice de rotation équivalente (figure 3) qui transforme les coordonnées d'un vecteur exprimé dans le référentiel terrestre au référentiel du verre.

$$
\left[\begin{array}{ccc}
1-2\left(q y^{2}-q z^{2}\right) & 2(q x . q y-q w \cdot q z) & 2(q x . q z+q w \cdot q y) \\
2(q x . q y+q w \cdot q z) & 1-2\left(q x^{2}-q z^{2}\right) & 2(q y \cdot q z-q w \cdot q x) \\
2(q x . q z-q w \cdot q y) & 2(q y \cdot q z+q w \cdot q x) & 1-2\left(q x^{2}-q y^{2}\right)
\end{array}\right]
$$

Figure 3. Matrice de rotation équivalente générée à partir du quaternion de pose

Nous avons appliqué cette matrice de rotation au vecteur vertical. Ensuite, nous avons déduit l'angle d'inclinaison en calculant le produit scalaire du vecteur résultant de la rotation et du vecteur vertical $(0$, $0,1)$.

\subsection{Détection des tremblements et caractérisation}

La détection et la caractérisation des tremblements sont basées sur un outil d'analyse spectrale fourni par le logiciel MatLab: Fast Fourier Transform (FFT). FFT met en évidence les composantes de fréquence d'un signal bruité dans le domaine temporel. Après avoir importé les données du gyroscope dans MatLab, nous appliquons une FFT pour chaque axe. Ensuite, nous calculons la densité spectrale de puissance (PSD) pour chaque axe. Le PSD est une mesure de l'énergie à diverses fréquences. Il décrit comment la puissance du signal est répartie sur la fréquence. Par la suite, nous effectuons manuellement l'analyse PSD avec les outils MatLab tels que le périodogramme et recherchons les pics de fréquence pour chaque échantillon. La valeur maximale de PSD correspond à la fréquence fondamentale du signal. Enfin, nous affichons un graphique avec les valeurs de fréquence et de puissance de la fréquence fondamentale. 


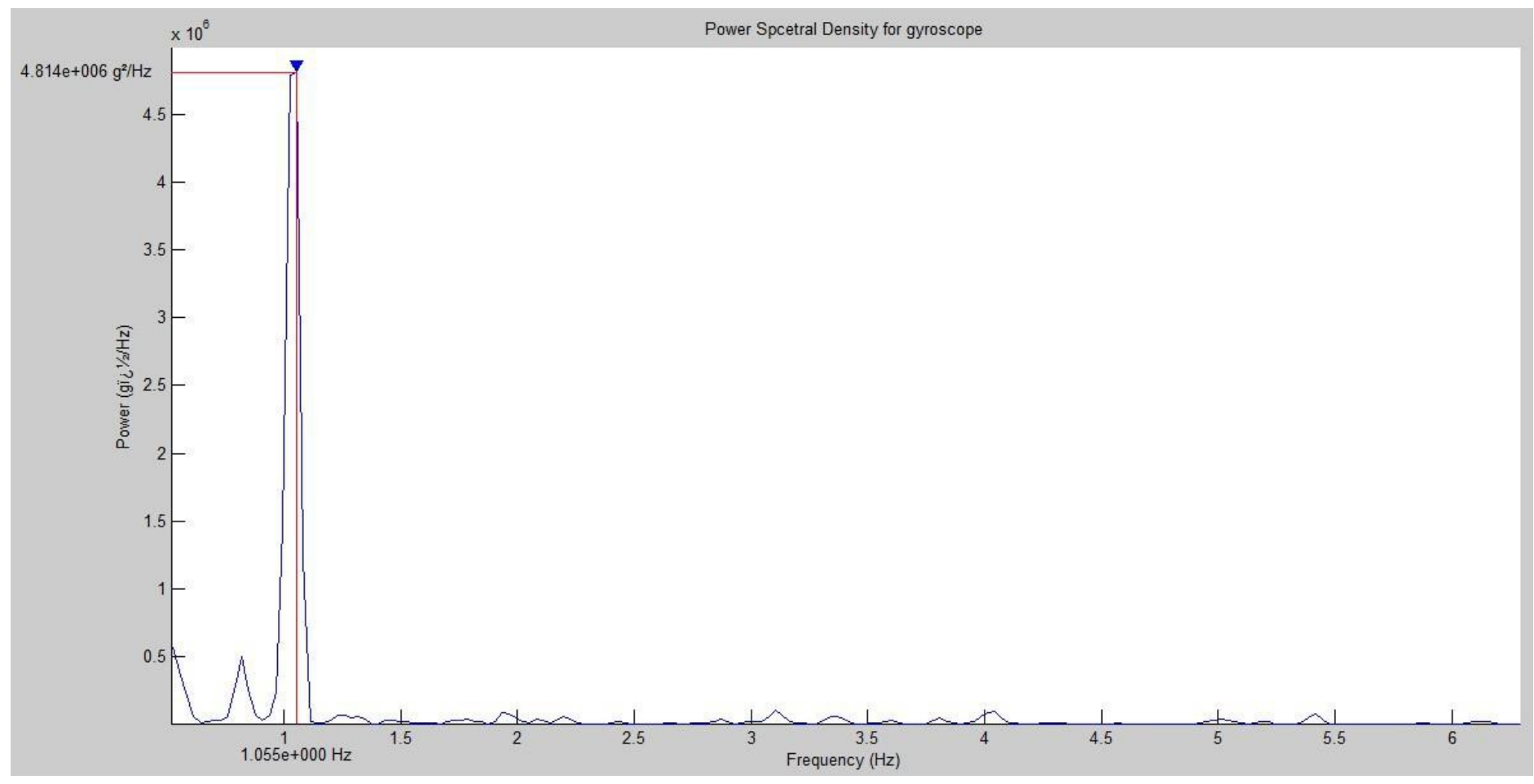

Figure 4. Exemple de détection de tremblement sur l'axe $Z$ du gyroscope

\section{Etude technique}

L'étude technique se concentre sur l'évaluation de la fiabilité et de la précision de la détection des tremblements. Cette information est très importante pour les thérapeutes afin de suivre les progrès des patients et de détecter certains problèmes de santé. Les autres traitements de données sont largement abordés dans la littérature (i.e. le suivi d'orientation et la détection basée sur NFC) [21], [22]. Ils ne sont donc pas abordés dans cet article.

\subsection{Protocole}

Pour générer des tremblements gyroscopiques contrôlés, un moteur pas à pas a été utilisé. Un potentiomètre a été ajouté sur le moteur pour calibrer la fréquence du tremblement et pour générer une fréquence précise. Lorsque le potentiomètre détecte une valeur minimale (ou maximale), la rotation est inversée. Le temps entre-deux minimums (ou maximums) permet de calculer la fréquence du moteur: $1000=\Delta \mathrm{t}(\Delta \mathrm{t}$ exprimé en $\mathrm{ms})$. Nous avons effectué trente mesures sur chaque axe pour cinq fréquences de tremblements différentes $(1,2,3,4,5 \mathrm{~Hz})$ selon les spécifications mises en évidence par Smaga et al. [19].

\subsection{Résultats}

Nous avons calculé les moyennes de chaque échantillon (1 à $5 \mathrm{~Hz}$ sur $\mathrm{X}$, Y et $\mathrm{Z}$ ) et calculé le pourcentage d'erreur. Ensuite, nous avons calculé le pourcentage d'erreur général pour chaque axe afin de le comparer au pourcentage d'erreur de la documentation.

\begin{tabular}{|c|c|c|c|c|c|c|}
\hline & $\mathbf{1 ~ H z}$ & $\mathbf{2 ~ H z}$ & $\mathbf{3 ~ H z}$ & $\mathbf{4 ~ H z}$ & $\mathbf{5 ~ H z}$ & Moyenne \\
\hline $\mathbf{X}$ & 3.12 & 4.00 & 3.55 & 3.96 & 3.71 & 3.66 \\
\hline $\mathbf{Y}$ & 3.32 & 4.00 & 3.55 & 3.86 & 3.69 & 3.68 \\
\hline $\mathbf{Z}$ & 2.93 & 4.00 & 3.61 & 3.80 & 3.77 & 3.66 \\
\hline
\end{tabular}

Tableau 2. Pourcentage d'erreur mesuré pour une spécification de $\pm 3 \%$ 
Le tableau adjacent (tableau 2) montre que le pourcentage d'erreur est indépendant de l'axe de rotation. De plus, l'IMU est conforme à la fiche technique qui spécifie une plage d'erreur de $3 \%$. La différence entre nos résultats et la fiche technique pourrait être réduite en augmentant la fréquence d'échantillonnage ou en utilisant un moteur à courant continu afin d'éviter les vibrations parasites.

\section{Etudes cliniques en prévision}

Basées sur ce prototype de SyMPATHy, deux études sont prévues avec les thérapeutes et les patients. Le premier objectif est d'avoir des retours afin d'améliorer le design et les fonctionnalités du verre. L'utilisabilité et l'acceptabilité de la plateforme seront également étudiées. Enfin, l'impact de SyMPATHy sur l'état de santé des patients devrait être évalué.

\subsection{Etudes avec les thérapeutes}

SyMPATHy fournit un grand nombre de données pertinentes sur l'activité du patient (enregistrements temporels, valeurs moyennes, etc.). Les données collectées doivent être affichées afin de fournir des informations utilisables pour les thérapeutes. Une étude avec ces derniers travaillant dans un centre de rééducation sera menée afin de créer un outil de visualisation répondant à leurs attentes. Cette étude devrait souligner les informations les plus importantes à afficher ainsi que la manière de les afficher. Plus fondamentalement, cette étude évaluera l'utilité des données récoltées par ce type de plate-forme pour suivre les AVQs.

\subsection{Etudes avec les patients}

Une autre étude avec des patients dans un centre de rééducation doit être conduite. L'objectif de cette étude est d'évaluer l'acceptabilité du verre dans le processus de rééducation. Tout d'abord, le verre (fonctionnalités et affichage) sera présenté aux patients. Ensuite, les patients devront utiliser le verre sans feedback. Après ça, les feedback seront activés. Durant chaque étape, l'acceptabilité et l'utilisabilité de SyMPATHy seront évaluées avec les patients et les thérapeutes. Les résultats de cette étude devraient mettre en évidence les fonctionnalités à améliorer et nous permettre d'identifier les faiblesses pouvant mener à un rejet de ce concept technologique [23]

\section{Conclusion}

Le prototype de verre intelligent SyMPATHy a été développé pour suivre l'activité des patients et les guider durant les AVQs. Les données collectées fournissent des informations pertinentes sur l'évolution de santé du patient et permettent au thérapeute d'adapter son programme de rééducation. Une étude technique a montré que la détection des tremblements avec la plate-forme SyMPATHy est fiable et précise. Enfin, deux études cliniques sont prévues afin d'améliorer les fonctionnalités du verre et de créer un outil de visualisation des données utilisable pour les thérapeutes ainsi que d'évaluer l'utilisabilité du verre pour les patients et les thérapeutes.

\section{Bibliographie}

[1] J. S. Kim, « Delayed onset hand tremor caused by cerebral infarction. », Stroke; a journal of cerebral circulation, vol. $23, n^{\circ} 2$, p. 292-294, 1992.

[2] S. Dethy, A. Luxen, L. M. Bidaut, et S. Goldman, « Hemibody tremor related to stroke. », vol. 24, n 12, p. 2094-2096, 1993.

[3] S. Pandian et K. N. Arya, « Stroke-related motor outcome measures: Do they quantify the neurophysiological aspects of upper extremity recovery? », vol. 18, $\mathrm{n}^{\circ} 3$, p. 412-423, 2014.

[4] N. Bagalkot, E. Nazzi, et T. Sokoler, « Facilitating continuity: exploring the role of digital technology in physical rehabilitation ", in Proceedings of the 6th Nordic Conference on Human-Computer Interaction: Extending Boundaries, 2010, p. 42-51.

(C) 2017 ISTE OpenScience - Published by ISTE Ltd.London, UK-openscience.frPage | 9 
[5] J. H. Carr, R. B. Shepherd, L. Nordholm, et D. Lynne, « Investigation of a new motor assessment scale for stroke patients », vol. $65, \mathrm{n}^{\circ} 2$, p. 175-180, 1985.

[6] A. R. Fugl-Meyer, L. Jääskö, I. Leyman, S. Olsson, et S. Steglind, « The post-stroke hemiplegic patient. 1. a method for evaluation of physical performance. », vol. 7, $\mathrm{n}^{\circ} 1, \mathrm{p} .13-31,1974$.

[7] G. D. Fulk et E. Sazonov, « Using sensors to measure activity in people with stroke », vol. 18, n 6, p. 746-757, 2011.

[8] M. Iosa et al., « Seven capital devices for the future of stroke rehabilitation », vol. 2012, 2012.

[9] A. Ferbert et M. Gerwig, « Tremor due to stroke », vol. 8, nº 2, p. 179-182, 1993.

[10] A. Handley, P. Medcalf, K. Hellier, et D. Dutta, « Movement disorders after stroke », vol. 38, n 3, p. 260-266, 2009.

[11] J. S. Kim, « Delayed onset mixed involuntary movements after thalamic stroke », vol. 124, n 2, p. 299-309, 2001.

[12] S. Lehericy et al., « Clinical characteristics and topography of lesions in movement disorders due to thalamic lesions $»$, vol. 57, nº 6, p. 1055-1066, 2001.

[13] B. Gialanella, R. Santoro, et C. Ferlucci, « Predicting outcome after stroke: the role of basic activities of daily living predicting outcome after stroke. », vol. 49, n 5, p. 629-637, 2013.

[14] A. A. Timmermans et al., « Arm and hand skills: training preferences after stroke », vol. 31, nº 16, p. 1344-1352, 2009.

[15] I. Aprile, M. Rabuffetti, L. Padua, E. Di Sipio, C. Simbolotti, et M. Ferrarin, « Kinematic analysis of the upper limb motor strategies in stroke patients as a tool towards advanced neurorehabilitation strategies: a preliminary study », vol. 2014, 2014.

[16] K. A. Sawner, J. M. LaVigne, et S. Brunnstrom, Brunnstrom's movement therapy in hemiplegia: a neurophysiological approach. Lippincott, 1992.

[17] M. A. Murphy, C. Willén, et K. S. Sunnerhagen, « Kinematic variables quantifying upper-extremity performance after stroke during reaching and drinking from a glass », vol. 25, $\mathrm{n}^{\circ} 1, \mathrm{p} .71-80,2011$.

[18] J. Schroeder et A. Fishbach, « How to motivate yourself and others? Intended and unintended consequences », vol. 35, p. $123-141,2015$.

[19] S. Smaga, «ESSENTIAL TREMOR », vol. 68, nº 8, 2003.

[20] L. Sartori, E. Straulino, U. Castiello, et A. Avenanti, « How objects are grasped: the interplay between affordances and end- goals », vol. 6, n 9, p. e25203, 2011.

[21] A. Kim et M. Golnaraghi, « A quaternion-based orientation estimation algorithm using an inertial measurement unit ", in Position Location and Navigation Symposium, 2004. PLANS 2004, 2004, p. 268-272.

[22] J. A. Rios et E. White, « Fusion filter algorithm enhancements for a MEMS GPS/IMU », p. 1-12, 2002.

[23] M. Anastassova, C. Mégard, et J.-M. Burkhardt, «Prototype evaluation and user-needs analysis in the early design of emerging technologies », in Human-computer interaction. Interaction design and usability, Springer, 2007, p. 383392. 